\title{
Mechanism of Action of the Benzimidazole Fungicide on Fusarium graminearum: Interfering with Polymerization of Monomeric Tubulin But Not Polymerized Microtubule
}

\author{
Yujun Zhou, Jianqiang Xu, Yuanye Zhu, Yabing Duan, and Mingguo Zhou
}

All authors: College of Plant Protection, Nanjing Agricultural University, Key Laboratory of Monitoring and Management of Crop Diseases and Pest Insects, Ministry of Agriculture, Nanjing, China; and second author: College of Forestry, Henan University of Science and Technology, Tianjing Rd No 70, 471003, Luoyang, People's Republic of China. Accepted for publication 5 March 2016.

\begin{abstract}
Zhou, Y., Xu, J., Zhu, Y., Duan, Y., and Zhou, M. 2016. Mechanism of action of the benzimidazole fungicide on Fusarium graminearum: Interfering with polymerization of monomeric tubulin but not polymerized microtubule. Phytopathology 106:807-813.

Tubulins are the proposed target of clinically relevant anticancer drugs, anthelmintic, and fungicide. $\beta_{2}$-tubulin of the plant pathogen Fusarium graminearum was considered as the target of benzimidazole compounds by homology modeling in our previous work. In this study, $\alpha_{1^{-}}, \alpha_{2^{-}}$, and $\beta_{2}$-tubulin of $F$. graminearum were produced in Escherichia coli. Three benzimidazole compounds (carbendazim, benomyl, and thiabendazole) interacted with the recombinant $\beta_{2}$-tubulin and reduced the maximum fluorescence intensity of $2 \mu \mathrm{M} \beta_{2}$-tubulin 47,50 , and $25 \%$, respectively, at saturation of compound-tubulin complexes. Furthermore, carbendazim

significantly inhibited the polymerization of $\alpha_{1}-/ \beta_{2}$-tubulins and $\alpha_{2}-/ \beta_{2^{-}}$ tubulins $90.9 \pm 0.4$ and $93.5 \pm 0.05 \%$, respectively, in vitro. A similar result appeared with benomyl on the polymerization of $\alpha_{1}-/ \beta_{2}$-tubulins and $\alpha_{2}-/ \beta_{2}$-tubulins at $89.9 \pm 0.1 \%$ and $92.6 \pm 1.2 \%$ inhibition ratios, respectively. In addition, thiabendazole inhibited $81.6 \pm 1 \%$ polymerization of $\alpha_{1}-/ \beta_{2}$-tubulins, whereas it had less effect on $\alpha_{2}-/ \beta_{2}$-tubulin polymerization, with $20.1 \pm 1.9 \%$ inhibition ratio. However, the three compounds cannot destabilize the polymerized microtubule. To illuminate the issue, mapping the carbendazim binding sites and $\beta / \alpha$ subunit interface on $\beta / \alpha$-tubulin complexes by homology modeling showed that the two domains were closed to each other. Understanding the nature of the interaction between benzimidazole compounds and $F$. graminearum tubulin is fundamental for the development of tubulin-specific antiF. graminearum compounds.
\end{abstract}

Benzimidazole derivatives are of wide interest because of their diverse biological activity and applications; they are remarkably effective compounds with respect to both their inhibitory activity and their favorable selectivity ratio (Ansari and Lal 2009; Hosamani and Shingalapur 2011; Navarrete-Vázquez et al. 2006). The benzimidazole ring shared by benzimidazole compounds displays an important heterocyclic pharmacophore in drug discovery. These compounds, carrying different substituents in the benzimidazole structure, are associated with a wide range of biological activities, including anticancer, antiviral, antibacterial, antifungal, antihelminthic, anti-inflammatory, antihistaminic, proton pump inhibitor, antioxidant, antihypertensive, and anticoagulant properties (Tunçbilek et al. 2009). Initial studies on the mechanism of action of the benzimidazoles focused on DNA and RNA synthesis. It soon became clear that the observed inhibition of DNA synthesis was a secondary effect, and that blockage of nuclear division was primarily responsible for this effect (Clemons and Sisler 1971; Davidse 1973; Hammerschlag and Sisler 1973). In arresting nuclear division of fungi, the benzimidazoles show a striking resemblance to the secondary plant metabolite colchicine, which disrupts mitosis and meiosis in animal and plant cells by inactivating the spindle. Biochemical studies on the mechanism of action of benzimidazole compounds have shown that the antimitotic action of MBC in fungi is probably mediated via binding to fungal tubulin (Davidse 1975). In addition, more evidence of

Corresponding author: M. Zhou; E-mail address: mgzhou@njau.edu.cn

Y. Zhou and J. Xu contributed equally to this work.

http://dx.doi.org/10.1094/PHYTO-08-15-0186-R

(c) 2016 The American Phytopathological Society the mechanism of action of the benzimidazoles on the fungi was derived from latter biochemical studies (Davidse 1987).

Benzimidazole derivatives play an important role in controlling various fungal pathogens. The most popular fungicide is carbendazim (Fig. 1). The fungicide is used to control plant diseases in cereal and fruit. Binding experiments using $\left[{ }^{14} \mathrm{C}\right]$ carbendazim and crude mycelial extract of three strains of Aspergillus nidulans indicated that binding to a cellular protein, with characteristics typical for tubulin, was involved in the mechanism of action of benzimidazole fungicides (Davidse and Flach 1977). Competitive inhibition of $\left[{ }^{14} \mathrm{C}\right]$ carbendazim binding by colchicine and nocodazole supported the idea that the carbendazim binding protein was fungal tubulin. Colchicine and nocodazole effectively inhibit microtubule assembly of tubulin from mammalian sources, whereas carbendazim only slightly affects this process (Friedman and Platzer 1978; Hoebeke et al. 1976). Assembly of yeast tubulin, however, is sensitive to both nocodazole and carbendazim. These in vitro studies strongly support the idea that the cytological effects of benzimidazoles in fungi are caused by an interference with the normal functioning of microtubules because benzimidazoles bind to tubulin. Interference with microtubule assembly in vivo has been convincingly demonstrated by Howard and Aist $(1977,1980)$, who studied the various effects of carbendazim on hyphal tip cells of Fusarium acuminatum with light and electron microscopy.

$F$. graminearum is a filamentous fungus that causes the serious infectious disease Fusarium head blight (FHB) on wheat, maize, and barley, and can cause severe yield and economic losses in these crops worldwide (Goswami and Kistler 2004). Benzimidazoles, including carbendazim, are a group of broad-spectrum, systemic fungicides which have been used to control FHB by interacting with $\beta$-tubulin and stopping hyphal growth (Hollomon et al. 1998). 
In recent studies from the authors' laboratory (Chen et al. 2008, 2009 ), point mutations in the $\beta_{2}$-tubulin gene at residues 167 (Phe to Tyr), 198 (Glu to Lys), and 200 (Phe to Tyr) were detected in benzimidazole-resistant field strains of $F$. graminearum. In addition, these sites constituted a hydrophobic pocket in $\beta_{2}$-tubulin by homology modeling, which identified the possible binding domain for benzimidazoles (Qiu et al. 2011). In addition, benomyl (Delp and Klopping 1968) (conversion products carbendazim and butyl iso-cyanate) and thiabendazole (Staron et al. 1964) were introduced as fungicides. Thiabendazole was also applied as anthelmintic (Brown et al. 1961). Both benomyl and thiabendazole belong to benzimidazole compounds.

Although the mechanisms of action of benzimidazoles on the fungi, parasites, and mammal cells have been extensively studied, most of the research was derived at the biochemical level but not the molecular level. The potency with which presumed antimicrotubule drugs inhibit microtubule assembly can be determined by in vitro assays using partially purified tubulin. In initial studies, only mammalian brain cells could be used as the tubulin source because of the high concentration of tubulin needed. The tubulin content was as high as $30 \%$ in porcine brain cells (Castoldi and Popov 2003). In contrast, the content of tubulin in fungal cells was below $1 \%$; a yield of tubulin at $0.5 \mathrm{mg} /$ liter was purified by overexpression of tubulin in A. nidulans, which was the first report of the purification of assembly-competent tubulin from a filamentous fungus (Yoon and Oakley 1995). Recently, one-step purification of assembly-competent tubulin from diverse eukaryotic sources was developed but purified tubulin was $0.1 \%$ of the total protein in yeast extract (Widlund et al. 2012). Furthermore, expression and purification of tubulin fused with maltose-binding protein in Escherichia coli have been reported but the recombinant tubulin cannot be polymerized in vitro (Hollomon et al. 1998). The purpose of this study was to overcome the difficulties in preparing sufficient quantities of fungal tubulin by purification of recombinant $\alpha$ - and $\beta_{2}$-tubulins of $F$. graminearum. Furthermore, effects of carbendazim, benomyl, and thiabendazole on the polymerization of $F$. graminearum tubulins were investigated using turbidity and homology modeling, providing more information of the mechanism of action of benzimidazoles. This work will promote structural and functional research into fungal tubulin. In future, the $F$. graminearum tubulins will be used as a tool in screening of antimitotic compounds.

\section{MATERIALS AND METHODS}

Strains and plasmids. F. graminearum strain 2021 (Chen et al. 2007), which was isolated from wheat in Zhejiang Province and sensitive to carbendazim, reserved in our lab, was used as the original strain to amplify the $\alpha$-tubulin genes. Plasmid $p E T 30 a^{+}$was purchased from Novagen (Merck). E. coli strain DH5a was used as the recipient for subcloning, and strain BL21 (DE3) pLysS was used for protein expression.

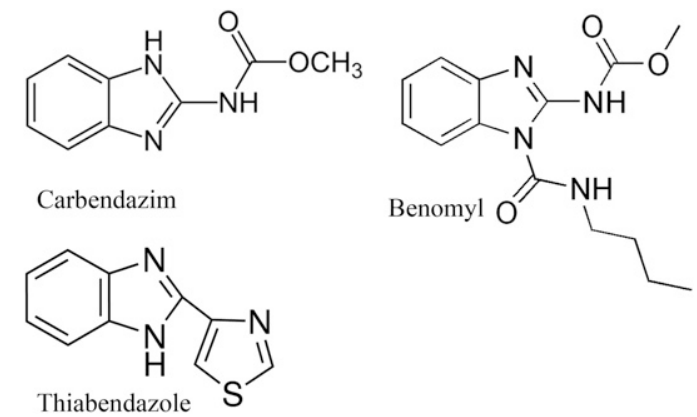

Fig. 1. Structures of benzimidazole compounds of carbendazim, benomyl, and thiabendazole.
Construction of expression plasmids. For preparation of RNA from vegetative hyphae, each strain was grown in liquid potato sucrose (Dingguo) for $36 \mathrm{~h}$. Total RNA was extracted using Trizol REAGENT (Invitrogen) following the manufacturer's protocol and was quantified by spectrophotometry at 260/280 $\mathrm{nm}$. The RNA was then used as the template for reverse-transcription polymerase chain reaction (RT-PCR). The $\alpha_{1}$-tubulin (FGSG_00639) and $\alpha_{2}$-tubulin (FGSG_00397) genes were amplified by PCR using the cDNA of $F$. graminearum. The primers (Table 1) were $\alpha_{1}$-30F: $5^{\prime}$-GCACATATGCGTGAGGTCATTAG-3' (the recognition site of $N d e \mathrm{I}$ is underlined); $\alpha_{1}-30 \mathrm{R}: 5^{\prime}$-GTAGAATTCCCGTACT CAGCCTCCAA-3' (the recognition site of EcoRI is underlined); $\alpha_{2}$-30F: 5'-CGCGGATCCATGAAGGGCGAGATTC-3' (the recognition site of Bam HI is underlined); and $\alpha_{2}-30 \mathrm{R}$ : 5'-CATGCGGCCGCC TAGTACTCGAGTTCCT-3' (the recognition site of $N o t \overline{\mathrm{I}}$ is underlined). The primers used to amplify the $\beta_{2}$-tubulin gene (FGSG_06611.3) were $\beta_{2}$-30F: 5'-CGTCATATGCGTGAGATTG-3' (the recognition site of NdeI is underlined) and $\beta_{2}$-30R: 5'-TACCATAAGCTTTCCACCC-3' (the recognition site of HindIII is underlined).

The PCR products were subcloned into the expression vector pET3Oa $a^{+}$to obtain expression constructs $p E T 30 a-\alpha_{1}$ tubulin, pET3Oa- $\alpha_{2}$ tubulin, and pET30a- $\beta_{2}$ tubulin. The recombinant expression plasmids were confirmed by restriction enzyme analysis and sequence.

Expression, refolding, and purification of recombinant F. graminearum $\boldsymbol{\alpha}_{1^{-}}, \boldsymbol{\alpha}_{2^{-}}$, and $\boldsymbol{\beta}_{2^{-}}$-tubulins. E. coli strain BL21 (DE3) pLysS was used as a host for expression of the target genes. The transformation of $p E T 30 a-\alpha_{1},-\alpha_{2}$, and $-\beta_{2}$ tubulin into E. coli was performed according to the manufacturer's instructions (Merck). The induction procedure for gene expression was as follows: $2 \mathrm{ml}$ of Luria-Bertani (LB) broth ( $10 \mathrm{~g}$ of tryptone, $5 \mathrm{~g}$ of yeast extract, and $10 \mathrm{~g}$ of $\mathrm{NaCl}$ per liter of $\mathrm{H}_{2} \mathrm{O}$ ) containing kanamycin at $50 \mu \mathrm{g} \mathrm{ml}^{-1}$ and chloramphenicol at $34 \mu \mathrm{g} \mathrm{ml}^{-1}$ was inoculated with a bacterial colony (BL21 [DE3] pLysS strain transformed with expression constructs) and incubated for $14 \mathrm{~h}$ at $225 \mathrm{rpm}$ and $37^{\circ} \mathrm{C}$. A 1-ml aliquot of the overnight culture was transferred into a flask containing $50 \mathrm{ml}$ of LB medium with the same antibiotic, and the flask was agitated at $225 \mathrm{rpm}$ at $37^{\circ} \mathrm{C}$ until the culture reached an optical density at $600 \mathrm{~nm}\left(\mathrm{OD}_{600}\right)=$ 0.4 to 0.6 . Isopropyl $\beta$-D-thiogalactoside was added to a final concentration of $1 \mathrm{mmol} \mathrm{liter}^{-1}$, and the culture was incubated at $225 \mathrm{rpm}$ and $37^{\circ} \mathrm{C}$ for $6 \mathrm{~h}$. After incubation, the bacterial cells were harvested by centrifugation at $6,000 \mathrm{rpm}$ for $10 \mathrm{~min}$ at $4^{\circ} \mathrm{C}$ and frozen at $-80^{\circ} \mathrm{C}$. A noninduced culture was used as a negative control.

$E$. coli cells overexpressing recombinant $F$. graminearum tubulins were resuspended in lysing buffer composed of $20 \mathrm{mM}$ Tris- $\mathrm{HCl}$ buffer (pH 8.0), $150 \mathrm{mM} \mathrm{NaCl}$, and 1\% (vol/vol) Triton X-100 and disrupted by ultrasonication. Cell-free extract was centrifuged at $12,000 \times g$ for $20 \mathrm{~min}$ at $4^{\circ} \mathrm{C}$ and the pellets (inclusion bodies) were refolded, as previously reported (Jang et al. 2008). The pellets obtained after sonication was dissolved in binding buffer $(6 \mathrm{M}$ guanidine hydrochloride, $20 \mathrm{mM}$ Tris, $300 \mathrm{mM} \mathrm{NaCl}, 20 \mathrm{mM}$ imidazole, and $1 \mathrm{mM} \beta$-mercaptoethanol, $\mathrm{pH} 8.0$ ) and the supernatant was collected by centrifugation at $12,000 \times g$ for $10 \mathrm{~min}$ at $4^{\circ} \mathrm{C}$. Supernatant containing tubulin was loaded onto a Ni-charged sepharose column preequilibrated with the binding buffer. The column was washed with binding buffer to base line, then with the

TABLE 1. Polymerase chain reaction primers used in this study ${ }^{\mathrm{a}}$

\begin{tabular}{lll}
\hline Primer & \multicolumn{1}{c}{ Sequence $\left(5^{\prime}\right.$ to $\left.3^{\prime}\right)$} & $\begin{array}{c}\text { Recognition } \\
\text { site }\end{array}$ \\
\hline$\alpha_{1}-30 \mathrm{~F}$ & GCACATATGCGTGAGGTCATTAG & NdeI \\
$\alpha_{1}-30 \mathrm{R}$ & GTAGAATTCCCGTACTCAGCCTCCAA & EcoRI \\
$\alpha_{2}-30 \mathrm{~F}$ & CGCGGATCCATGAAGGGCGAGATTC & Bam $\mathrm{HI}$ \\
$\alpha_{2}-30 \mathrm{R}$ & CATGCGGCCGCCTAGTACTCGAGTTCCT & Not I \\
$\beta_{2}-30 \mathrm{~F}$ & CGTCATATGCGTGAGATTG & NdeI \\
$\beta_{2}-30 \mathrm{R}$ & TACCATAAGCTTTCCACCC & HindIII \\
\hline
\end{tabular}

a Recognition sites are underlined. 
washing buffer (6 M urea, $20 \mathrm{mM}$ Tris, $300 \mathrm{mM} \mathrm{NaCl}, 40 \mathrm{mM}$ imidazole, and $1 \mathrm{mM} \beta$-mercaptoethanol, $\mathrm{pH}$ 8.0). The bound protein was refolded on-column using a decreasing gradient of urea ( 6 to $0 \mathrm{M}$ ), starting with the washing buffer and finishing with the refolding buffer (20 $\mathrm{mM}$ Tris, $300 \mathrm{mM} \mathrm{NaCl}, 20 \mathrm{mM}$ imidazole, and $1 \mathrm{mM} \beta$-mercaptoethanol, $\mathrm{pH}$ 8.0). The refolded protein was eluted using elution buffer ( $20 \mathrm{mM}$ Tris, $300 \mathrm{mM} \mathrm{NaCl}, 0.5 \mathrm{M}$ imidazole, and $1 \mathrm{mM} \beta$-mercaptoethanol, $\mathrm{pH} 8.0)$. Fractions containing the target protein were dialyzed against polymerization buffer $(0.1 \mathrm{mM}$ Pipes [pH 6.8], $0.1 \mathrm{mM} \mathrm{NaOH}, 1 \mathrm{mM}$ EGTA, $1 \mathrm{mM} \mathrm{MgSO}_{4}$, and $5 \%$ glycerol), concentrated, quantified using the Bradford method (Bradford 1976), and stored at $-80^{\circ} \mathrm{C}$. The purity of tubulin was assessed by sodium dodecyl sulfate polyacrylamide gel electrophoresis and the integrity of tubulin was confirmed by Western blot using an anti-His antibody (Zen BioScience), as previously reported (Jang et al. 2008).

Drug-tubulin binding assay. The interaction of each test compound (carbendazim, benomyl, and thiabendazole) with recombinant $F$. graminearum $\beta_{2}$-tubulin were measured as the amount of reduction in the intrinsic fluorescence of tryptophan residues following incubation with each compound (Eftink and Ghiron 1981). Equilibrium binding was determined by measuring the reduction in fluorescence intensity of recombinant $F$. graminearum $\beta_{2}$-tubulin following incubation with a range of concentrations of each compound. Each concentration of compound $(2,8,20$, and $40 \mu \mathrm{M}$ ) was incubated with $2 \mu \mathrm{M}$ tubulin for $30 \mathrm{~min}$ at $37^{\circ} \mathrm{C}$ and fluorescence was immediately measured with excitation of $280 \mathrm{~nm}$ and emission of $340 \mathrm{~nm}$ (Giles et al. 2009; MacDonald et al. 2004). Emission spectra were corrected for inner filter effects due to drug addition according to previously published equation (Mertens and Kägi 1979) $F($ corr $)=F(o b s) \times \operatorname{antilog}(A x+A m) / 2$, where $F(c o r r)$ is the corrected fluorescence intensity, $F(o b s)$ is the observed intensity, and $A x$ and $A m$ are the measured absorbance at the wavelengths of excitation and emission, respectively. The corrected fluorescence intensity values from three independent tests were then used to determine the percent reduction of fluorescence intensity.

Tubulin polymerization in vitro. Tubulin polymerizations were assayed at $\mathrm{A}_{350}$ using a Molecular Devices VersaMax microplate reader equipped with temperature controllers. The tubulin monomers were polymerized using a standard polymerization assay, as previously reported (Dong et al. 2004; Jang et al. 2008; Koo et al. 2009b).

The reaction mixture included $4 \mu \mathrm{M} \beta_{2}$-tubulin plus $4 \mu \mathrm{M} \alpha_{1}$ or $\alpha_{2}$-tubulin isotypes mixed with Pipes buffer to give a final concentration of $2 \mu \mathrm{M}$ for each tubulin isotype. Then, guanosine-5' triphosphate, disodium salt (GTP) (Dingguo) was added to a final concentration $2 \mathrm{mM}$ in the total volume (Oxberry et al. 2001). OD value was measured at $350 \mathrm{~nm}$ at $37^{\circ} \mathrm{C}$, every $5 \mathrm{~min}$ for $1 \mathrm{~h}$ (Koo et al. 2009a, 2010). At this wavelength (350 nm), scattering of light produced during the polymerization process causes an increase in absorbance proportional to the formation of tubulin aggregates.

Using this polymerization procedure, the effects of carbendazim, benomyl, and thiabendazole at a final concentration of $20 \mu \mathrm{M}$ (which had been dissolved in dimethyl sulfoxide) on $\alpha$ - and $\beta_{2}$-tubulin polymerization were assessed. Compounds were added to the polymerization mixture before and after incubation at $37^{\circ} \mathrm{C}$ for $1 \mathrm{~h}$ to investigate the effect of compounds on monomeric and polymerized tubulins, respectively. The degree of polymerization was assessed by subtracting the initial absorbance reading from the final reading obtained after $1 \mathrm{~h}$. The inhibition ratios of benzimidazole compounds on polymerization were calculated using the formula inhibition ratio $=(P a-P b / P a) \times 100$, where $P a$ was the polymerization degree of $\alpha$-plus $\beta_{2}$-tubulins without adding benzimidazole compounds and $P b$ was the polymerization degree after adding the compounds. All tests were repeated three times. The average inhibition ratios of benzimidazole compounds on polymerization are the means of the three experiments, and significant differences among the experiments were calculated using Fisher's least significant difference test.

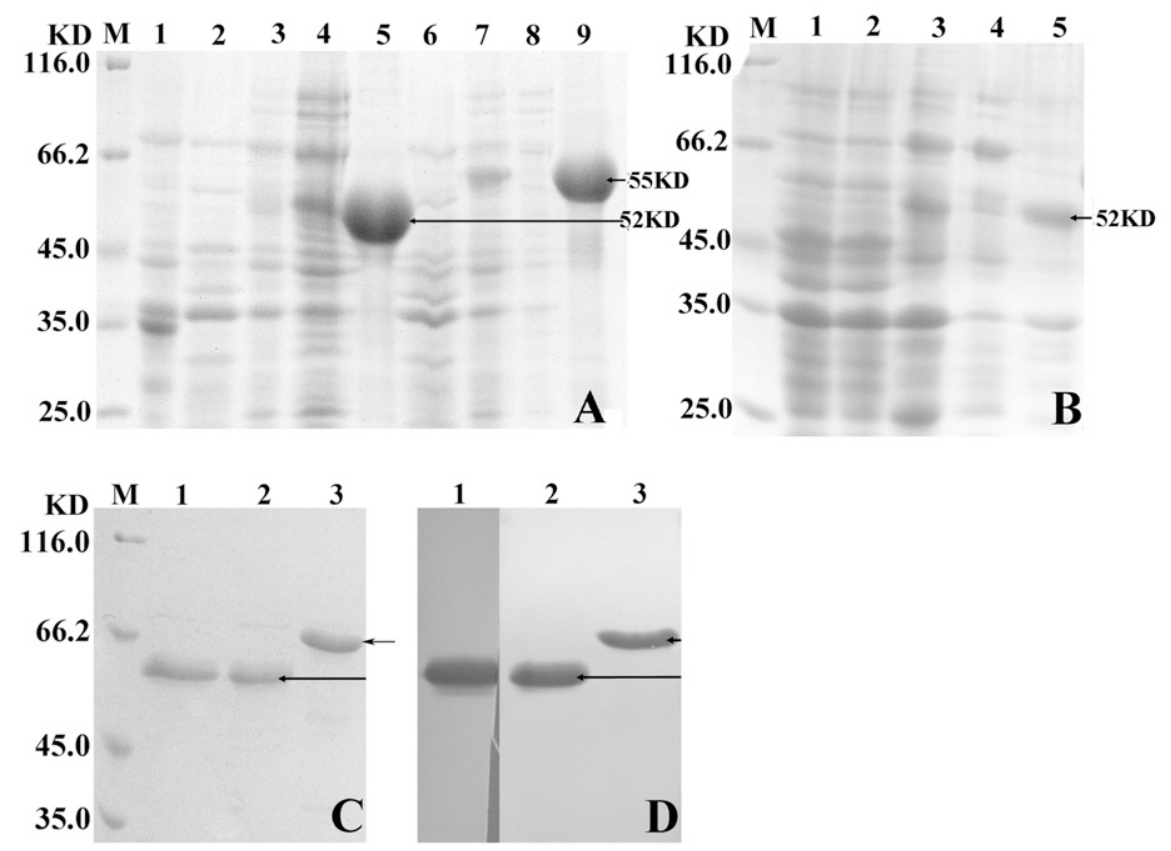

Fig. 2. Production of recombinant $\alpha_{1^{-}}, \alpha_{2^{-}}$, and $\beta_{2^{-}}$tubulins in Escherichia coli. A, Lane 1: total protein of BL21 (DE3) pLysS transformed with $p E T 30 a^{+}$. Lanes 2 to 5: samples of $\alpha_{1}$-tubulin; lanes 2 and 3: total protein without and with isopropyl $\beta$-D-thiogalactoside (IPTG) induction, respectively; lanes 4 and 5: supernatant and precipitation fraction after induction, respectively. Lanes 6 to 9: samples of $\alpha_{2}$-tubulin; lanes 6 and 7: total protein without and with IPTG induction, respectively; lanes 8 and 9: supernatant and precipitation fraction after induction, respectively. B, Lane 1: total protein of BL21 (DE3) pLysS transformed with $\mathrm{pET3Oa}^{+}$. Lanes 2 to 5: samples of $\beta_{2}$-tubulin; lanes 2 and 3: total protein without and with IPTG induction, respectively; lanes 4 and 5: supernatant and precipitation fraction after induction, respectively. C, Lanes 1 to 3: purified $\alpha_{1^{-}}, \alpha_{2^{-}}$, and $\beta_{2^{-}}$tubulins, respectively. D, Lanes 1 to 3: Western blot analysis of

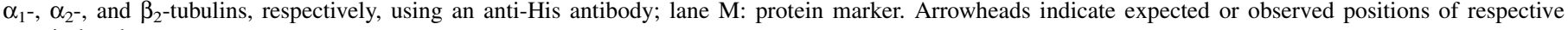
protein bands. 
Homology modeling of $F$. graminearum $\alpha$ - and $\beta_{2}-$ tubulins. The homology model of $F$. graminearum $\alpha-$ and $\beta_{2^{-}}$ tubulins was constructed using the online server at I-TASSER (Roy et al. 2010; Zhang 2008). Visualization and display of the amino acid residues on the homology structure were carried out using the PyMOL Molecular Graphics System (version 1.5.0.4; Schrödinger, LLC).

\section{RESULTS}

Expression, refolding, and purification of recombinant F. graminearum tubulins. The $\alpha_{1^{-}}, \alpha_{2^{-}}$, and $\beta_{2}$-tubulins were inserted into expression vector $p E T 30 a^{+}$and confirmed by double digestion reaction and sequencing (data not shown). All the constructs were transformed into E. coli strain BL21 (DE3) pLysS for protein expression. All the recombinant tubulins $\left(\alpha_{1^{-}}, \alpha_{2^{-}}\right.$, and $\beta_{2}$-tubulins) were expressed as inclusion bodies (Fig. 2A and B) and refolded on the columns (see Materials and Methods). The yields of

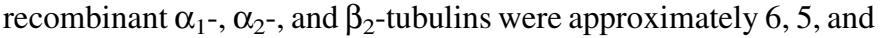
$3 \mathrm{mg} /$ liter, respectively. In addition, the purified recombinant $\alpha_{1^{-}}$, $\alpha_{2^{-}}$, and $\beta_{2^{-}}$-tubulins were above $90 \%$ pure (Fig. 2C). Western blot was performed on purified fusion-protein detected bands of the expected sizes for each recombinant protein, and indicated that the structural integrity of the tubulin fusion proteins was preserved throughout the expression and purification processes (Fig. 2D).

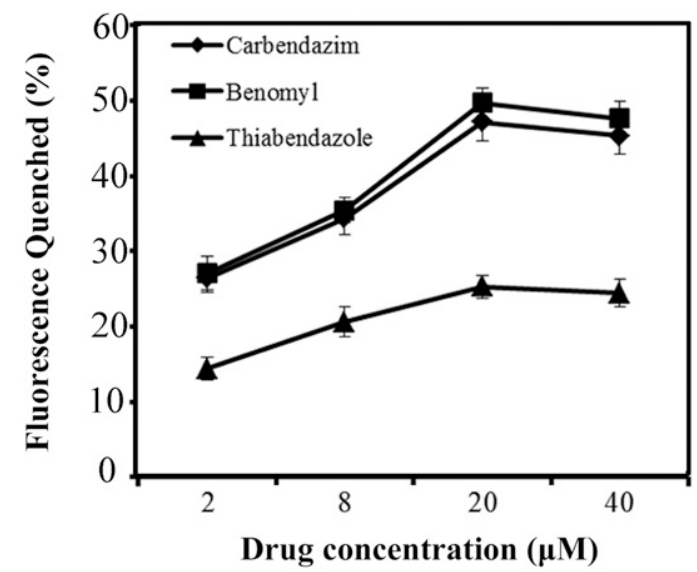

Fig. 3. Equilibrium binding of test compounds with recombinant Fusarium graminearum $\beta_{2}$-tubulin; the graph illustrates the proportion of tubulin fluorescence that was quenched following the addition of increasing concentrations of carbendazim, benomyl, and thiabendazole. Line bars in each point denote standard errors of three experiments.
Drug-tubulin binding assay. All three of the benzimidazole compounds interacted with recombinant $F$. graminearum $\beta_{2}$-tubulin and quenched fluorescence in a concentration-dependent manner (Fig. 3). Saturation of compound-tubulin complexes was achieved at concentrations of approximately $20 \mu \mathrm{M}$, as demonstrated by the plateau in the curves. A return in fluorescence occurred following saturation point due to the high concentrations of compounds exhibiting strong intrinsic fluorescence or conformational changes occurring in $\beta_{2}$-tubulin, causing dissociation of compounds. Carbendazim and benomyl at $20 \mu \mathrm{M}$ induced high reductions in fluorescence intensity of $\beta_{2}$-tubulin (47 and $50 \%$, respectively). In contrast, thiabendazole induced lower reductions in fluorescence intensity of $\beta_{2}$-tubulin (25\%).

Effects of carbendazim on $F$. graminearum tubulin polymerization in vitro. $F$. graminearum $\beta_{2}$-tubulin was proposed as the target for benzimidazole fungicides but how the drug interferes with tubulin polymerization was not clear. Each tubulin subunit $(2 \mu \mathrm{M}$ $\alpha_{1}-/ \beta_{2}$-tubulin and $2 \mu \mathrm{M} \alpha_{2}-/ \beta_{2}$-tubulin) was polymerized in the presence of $2 \mathrm{mM}$ GTP for $1 \mathrm{~h}$ at $37^{\circ} \mathrm{C}$ (Fig. 4). In addition, increasing $\mathrm{A}_{350}$ of $\alpha_{1}-/ \beta_{2}$-tubulin was 0.14 while that of $\alpha_{2}-/ \beta_{2}$-tubulin was 0.26 , which indicated that $\beta_{2}$-tubulin preferred binding to $\alpha_{2^{-}}$ tubulin rather than $\alpha_{1}$-tubulin.

To investigate the effects of carbendazim on tubulin polymerization in vitro, $20 \mu \mathrm{M}$ carbendazim was added to the polymerization mixture containing $\alpha$-plus $\beta_{2}$-tubulins, and the $A_{350}$ was measured after adding $2 \mathrm{mM}$ GTP to initiate the polymerization. The polymerizations for $\alpha_{1} / \beta_{2}$-tubulins and $\alpha_{2} / \beta_{2}$-tubulins both were significantly suppressed by the carbendazim at 91 and $93 \%$, respectively (Fig. 4A and B). However, almost no depolymerization was observed when $\alpha$-plus $\beta_{2}$-tubulins $\left(\alpha_{1} / \beta_{2}\right.$-tubulins and $\alpha_{2} / \beta_{2}$-tubulins) were polymerized for $1 \mathrm{~h}$ at $37^{\circ} \mathrm{C}$ followed by adding $20 \mu \mathrm{M}$ carbendazim ( 2 and $5 \%$ depolymerization ratios of $\alpha_{1} / \beta_{2}$-tubulins and $\alpha_{2} / \beta_{2}$-tubulins, respectively), which indicated that the polymerized tubulins were not affected by the carbendazim (Fig. 4A and B). In addition, in the absence of carbendazim, increasing $A_{350}$ of $\alpha_{1} / \beta_{2}$-tubulin was 0.14 while that of $\alpha_{2} / \beta_{2}$-tubulin was 0.26 , which indicated that $\beta_{2}$-tubulin preferred binding to $\alpha_{2}$-tubulin rather than $\alpha_{1}$-tubulin.

Inhibitions of benzimidazole compounds on $\boldsymbol{F}$. graminearum tubulin polymerization in vitro. To study whether some other benzimidazole compounds (benomyl and thiabendazole) had a similar mechanism of action on the $F$. graminearum tubulin polymerization, benomyl and thiabendazole were tested. Similarly to carbendazim, benomyl and thiabendazole did not have the ability to depolymerize polymerized tubulins $\left(\alpha_{1} / \beta_{2}\right.$-tubulins and $\alpha_{2} / \beta_{2}$-tubulins $)$ in vitro (data not shown). Furthermore, $20 \mu \mathrm{M}$ carbendazim could cause a significant decrease in the amount of $\alpha_{1} / \beta_{2}$-tubulin and $\alpha_{2} / \beta_{2}$-tubulin polymerization mixtures $(90.9 \pm 0.4$ and $93.5 \pm 0.05 \%$ inhibition ratios, respectively) (Table 2). The similar result also appeared with
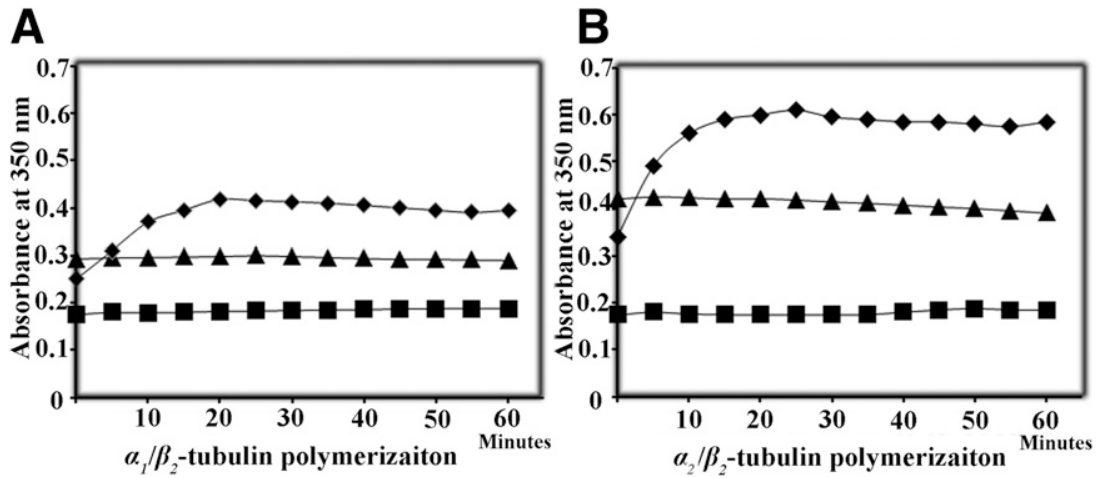

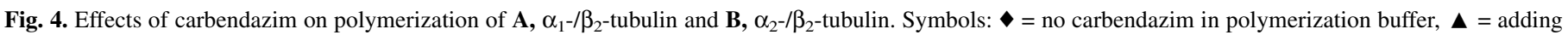

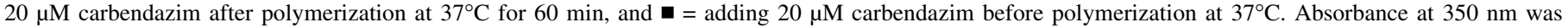
determined for $60 \mathrm{~min}$. Points $=$ means of triplicate measurements at each time point. 
the adding of $20 \mu \mathrm{M}$ benomyl in the $\alpha_{1} / \beta_{2}$-tubulin and $\alpha_{2} / \beta_{2}$-tubulin polymerization mixtures $(89.9 \pm 0.1$ and $92.6 \pm 1.2 \%$ inhibition ratios, respectively) (Table 2). In addition, thiabendazole inhibited the polymerization of $\alpha_{1} / \beta_{2}$-tubulin polymerization greatly (81.6 $\pm 1 \%$ inhibition ratio), whereas it had less effect on $\alpha_{1} / \beta_{2}$-tubulin polymerization $(20.1 \pm 1.9 \%$ inhibition ratio) (Table 2$)$. Thiabendazole had lower inhibition ratios of $\alpha_{2}$-/ $\beta_{2}$-tubulin polymerization than carbendazim and benomyl. In addition, the concentration resulting in $50 \%$ inhibition of mycelial growth $\left(\mathrm{EC}_{50}\right)$ of the three compounds for F. graminearum were $2.46,2.10$, and $5.61 \mu \mathrm{M}$, respectively, which were converted from published data of $\mathrm{EC}_{50}\left(\mu \mathrm{g} \mathrm{ml}{ }^{-1}\right)$ dividing by each molecular weight. The inhibition ratio and $\mathrm{EC}_{50}(\mu \mathrm{M})$ of the three compounds ranked carbendazim $\approx$ benomyl $<$ thiabendazole. This result indicated that $\mathrm{EC}_{50}\left(\mu \mathrm{g} \mathrm{ml}^{-1}\right)$ of the three compounds was negatively correlated with the inhibition ratios of $F$. graminearum $\alpha$-plus $\beta_{2}$-tubulin polymerizations. Furthermore, these results suggest that the interference of the three compounds with tubulin polymerization was the main mechanism of action.

Mapping the carbendazim binding sites and $\beta_{2} / \alpha$ subunit interface on $\boldsymbol{\beta}_{2} / \boldsymbol{\alpha}$-tubulin complexes. To illuminate why the benzimidazole compounds inhibit polymerization of the monomeric tubulins but not the polymerized microtubule, we

TABLE 2. Effect of benzimidazole compounds on the Fusarium graminearum and tubulin polymerization in vitro ${ }^{a}$

\begin{tabular}{lccc}
\hline Benzimidazole & $\begin{array}{c}\text { Inhibition ratio of } \\
\alpha_{1} \text { - and } \beta_{2} \text {-tubulin } \\
\text { polymerization }(\%)\end{array}$ & $\begin{array}{c}\text { Inhibition ratio of } \\
\alpha_{2^{-}} \text {and } \beta_{2} \text {-tubulin } \\
\text { polymerization }(\%)\end{array}$ & $\mathrm{EC}_{50}(\mu \mathrm{M})$ \\
\hline Carbendazim & $90.9 \pm 0.4$ & $93.5 \pm 0.05$ & 2.46 \\
Benomyl & $89.9 \pm 0.1$ & $92.6 \pm 1.2$ & 2.10 \\
Thiabendazole & $81.6 \pm 1.0$ & $20.1 \pm 1.9$ & 5.61 \\
\hline
\end{tabular}

a Inhibition ratios were measured by adding $20 \mu \mathrm{M}$ compounds followed by polymerizing at $37^{\circ} \mathrm{C}$. Values are means of three experiments and differences among the experiments were not significant (i.e., $P>0.05$, Fisher's least significant difference test). $\mathrm{EC}_{50}$ indicates the concentration of compound resulting in $50 \%$ inhibition of mycelial growth. $\mathrm{EC}_{50}(\mu \mathrm{M})$ were converted from data of $\mathrm{EC}_{50}\left(\mu \mathrm{g} \mathrm{ml}^{-1}\right)$ published in a previous report (Qiu et al. 2011). built two three-dimensional homology models of $F$. graminearum $\alpha_{1}-/ \beta_{2}$-tubulin and $\alpha_{2}-/ \beta_{2}$-tubulin protein, respectively, using an online platform for protein structure and function predictions, I-TASSER, based on multiple threading alignments. Superposition of homology structures of $F$. graminearum $\alpha_{1}-/ \beta_{2}$-tubulin (yellow $/$ red) dimer and $\alpha_{2}-/ \beta_{2}$-tubulin (yellow/red) dimer on the crystal structure of Saccharomyces cerevisiae $\alpha-1-/ \beta$-tubulin (blue/green) dimer (PDB accession 4FFB), respectively, showed a remarkable similarity of overall structure-folding between the two structures (Fig. 5A and B). Carbendazim binding pocket (cyan), consisting of amino acid positions $165,167,198$, and 200 , and the $\beta / \alpha$-tubulin interface (blue), consisting of amino acid positions 2, 131, 245 to $247,251,256,258$ to 260,322 to 324,327 , and 347 to 351 , were shown on $F$. graminearum $\beta_{2}$-tubulin (Fig. 5C and D).

\section{DISCUSSION}

Microtubules are built from $\alpha \beta$-tubulin heterodimers, which are targets of many antitumor drugs, anthelminthics, and fungicides. Attempts to understand the molecular basis of mechanism of benzimidazole reagents, related to $\beta_{2}$-tubulin in $F$. graminearum, have been hampered by the inability to purify tubulin from filamentous fungi on a large scale. In other ways, the resulting preparations (tubulins) from natural tissues were contaminated by microtubule-associated proteins and microtubule motor proteins; in addition, it was difficult to separate different tubulin subtypes due to their similar physico-chemical properties. In the present study, by using the $E$. coli expression system, we were able to produce enough $F$. graminearum $\alpha_{1^{-}}, \alpha_{2^{-}}$, and $\beta_{2}$-tubulins (recombinant tubulins at 6,5 , and $3 \mathrm{mg} /$ liter, respectively) with high purity $(>90 \%)$ and free of microtubule-associated proteins. In contrast, the best yield of tubulin purified from fungi in the literature was $<1.0 \mathrm{mg} /$ liter (Yoon and Oakley 1995). In our study, the recombinant tubulins mainly formed inclusion bodies which were consistent with previous reports of tubulins from other species expressed in E. coli (Blackhall et al. 2006; Koo et al. 2009a; Lubega et al. 1993). The recombinant tubulins of $F$. graminearum were refolded in column and demonstrated to have the biological function of polymerizing into a
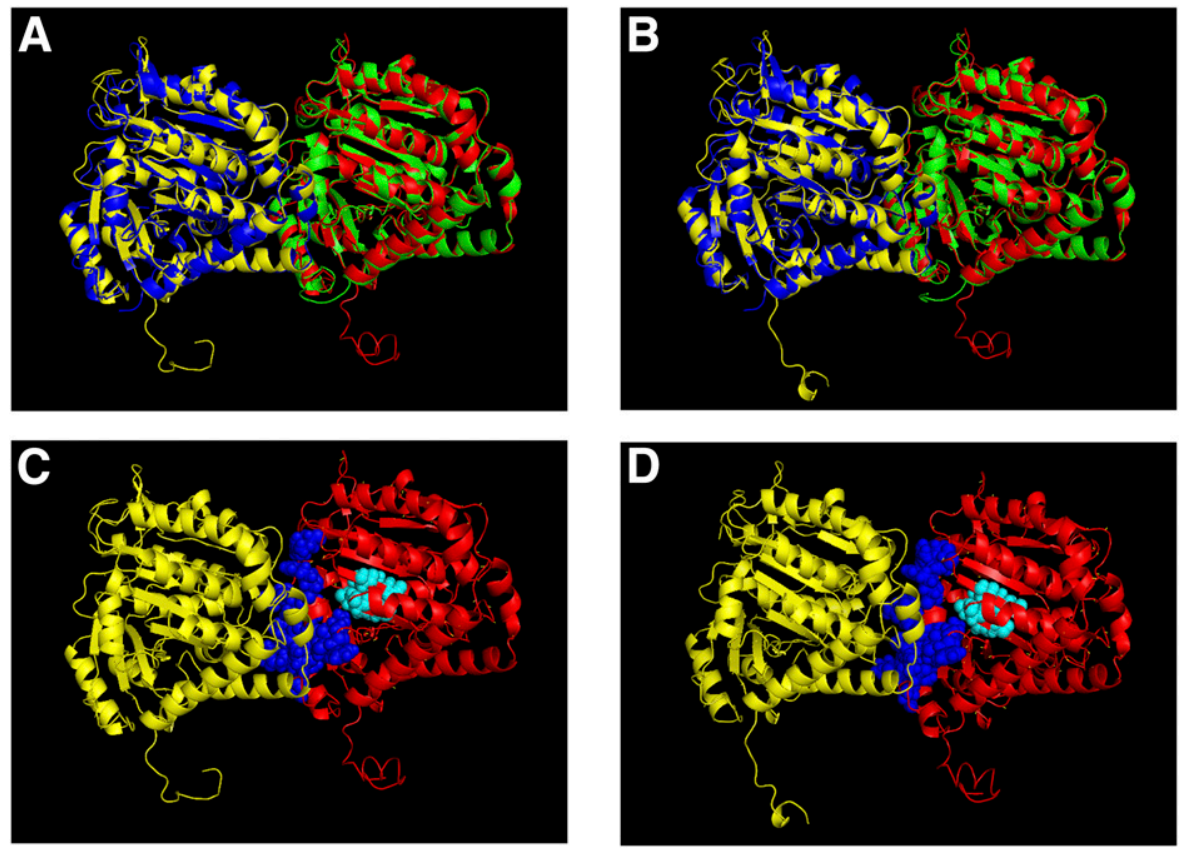

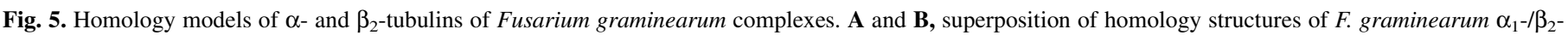

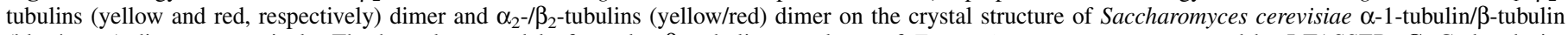

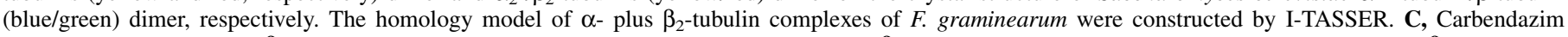

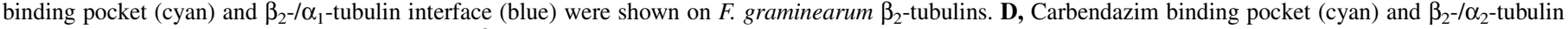
interface (blue) were shown on $F$. graminearum $\beta_{2}$-tubulins (red). 
microtubule. Although it is desirable to obtain pure native tubulin protein for characterization studies, refolded tubulins have been applied to polymerization (Koo et al. 2009a) and drug-tubulin interaction (Lubega et al. 1993) in early research.

Carbendazim has been proposed to bind to $\beta_{2}$-tubulin of F. graminearum using homology modeling (Qiu et al. 2011), while no evidence of the carbendazim-tubulin physical interaction was raised. In this study, interaction between three benzimidazole compounds with $\beta_{2}$-tubulin of $F$. graminearum was demonstrated by fluorescence quenching (Fig. 3). This result indicated that the $\beta$-tubulin was the binding site for benzimidazole, which has been reported in fungal and invertebrate hosts. Furthermore, the fluorescence quenching ratios of the three benzimidazole compounds (carbendazim, benomyl, and thiabendazole) to $\beta_{2}$-tubulin was negatively correlated with the $\mathrm{EC}_{50}$ values $(2.46,2.1$, and $5.61 \mu \mathrm{M}$, respectively), indicating that the binding affinity between the drug and $\beta_{2}$-tubulin may be the main mechanism of the action of benzimidazole compounds. In addition, this result was consistent with previous reports (Davidse 1986; Davidse and Flach 1977; Hollomon et al. 1998). Point mutation in $\beta$-tubulin can dramatically reduce carbendazim sensitivity in $F$. graminearum, which also supports the conclusion above (Qiu et al. 2011).

Studies with the classical antimitotic agent colchicine have shown that this compound binds to tubulin prior to its polymerization (Sackett and Varma 1993; Uppuluri et al. 1993). Inhibition of microtubule assembly appears to be achieved through addition of colchicine-bearing tubulin to the end of a growing microtubule and loss of the ability of these subunits to accept other tubulin molecules for further microtubule growth. Data obtained from fluorescence spectroscopy and labeling experiments have indicated that the colchicine binding site is on the $\beta$-tubulin monomer, obviously close to the $\alpha / \beta$ subunit interface (Uppuluri et al. 1993). In this study, the mechanism underlying the action of the benzimidazole compounds against $F$. graminearum was investigated using turbidimetric methods, as previously reports (Fourest-Lieuvin 2006; Minoura et al. 2013). In the absence of carbendazim, the $A_{350}$ of polymerization mixtures increased from 0.25 to 0.4 and 0.34 to 0.6 , indicating the polymerization of $\alpha_{1}-/ \beta_{2}$-tubulin and $\alpha_{2}-/ \beta_{2}$-tubulin, respectively (Fig. 4). This result indicated that $\beta_{2}$-tubulin preferred binding to $\alpha_{2}$-tubulin rather than $\alpha_{1}$-tubulin. By contrast, tiny changes of $A_{350}$ of polymerization mixtures of $\alpha_{1}-/ \beta_{2}$-tubulin and $\alpha_{2}-/ \beta_{2}$-tubulin were observed if carbendazim was added into the mixtures before the polymerization; this result indicated that the carbendazim significantly inhibited the polymerization of tubulins. This result was consistent with the mechanism of the action of colchicine. Furthermore, in order to investigate whether carbendazim interfered with microtubule dynamics by directly destabilizing the microtubule, polymerized tubulins were treated with carbendazim and no changes of $A_{350}$ of polymerization mixtures of $\alpha_{1}-/ \beta_{2}$-tubulin and $\alpha_{2}-/ \beta_{2}$-tubulin were observed. This result indicated that carbendazim cannot affect the assembled microtubule. Why would the benzimidazole compounds inhibit polymerization of the monomeric tubulins but not the polymerized microtubule? The carbendazim binding pocket in $\beta_{2}$-tubulin of $F$. graminearum, consisting of amino acids at positions $165,167,198$, and 200, was close to the $\alpha_{1} / \beta_{2^{-}}$and $\alpha_{2} / \beta_{2}$-tubulin subunit interfaces by homology modeling (Fig. 5). In addition, the carbendazim binding pocket was located inside the $\beta_{2}$-tubulin structure. Based on these results, we can assume that formation of the drug- $\beta$ tubulin complex resulted in a conformation change in the $\alpha / \beta_{2}$ subunit interface followed by loss of the ability of these subunits to accept other tubulin molecules for further microtubule growth. In contrast, polymerized microtubule may have a more compact structure which destroyed the accessibility of the drug to tubulins.

The mechanism underlying the activity of the benzimidazole compounds against $F$. graminearum was investigated in this study. The inhibition ratios of three benzimidazole compounds (carbendazim, benomyl, and thiabendazole) on the polymerization of $\alpha$ - plus $\beta_{2}$-tubulins were determined and the correlation of inhibition ratios between $\mathrm{EC}_{50}$ of compounds for $F$. graminearum were analyzed (Table 2). Inhibition ratio of $20 \mu \mathrm{M}$ carbendazim on $\alpha_{1^{-}}$and $\beta_{2}$-tubulin polymerization and $\alpha_{2}$-and $\beta_{2}$-tubulin polymerization were $90.9 \pm 0.4$ and $93.5 \pm 0.05 \%$, respectively. Similarly, the inhibition ratios of $20 \mu \mathrm{M}$ benomyl on $\alpha_{1}$-/ $\beta_{2}$-tubulin polymerization and $\alpha_{2}-/ \beta_{2}$-tubulin polymerization were $89.9 \pm 0.1$ and $92.6 \pm 1.2 \%$, respectively. Therefore, carbendazim has almost the same inhibition ratio as benomyl on tubulin polymerizations. This result can be explained because it is well established that, in either aqueous solutions or soil, benomyl (one molecular) rapidly degrades to its breakdown product carbendazim (one molecular) (Chiba and Cherniak 1978; Tang et al. 1992). In addition, this speculation was confirmed by the similar $\mathrm{EC}_{50}$ of carbendazim and benomyl on $F$. graminearum ( 2.46 and $2.10 \mu \mathrm{M}$, respectively). Butyl iso-cyanate (the other breakdown product of benomyl) also contributed to the drug function in vivo (Twu and Wold 1973). However, inhibition ratios of $20 \mu \mathrm{M}$ thiabendazole on $\alpha_{1}-/ \beta_{2^{-}}$ tubulin polymerization and $\alpha_{2}-/ \beta_{2}$-tubulin polymerization were $81.6 \pm 1$ and $20.1 \pm 1.9 \%$, respectively, which were remarkably lower than that of carbendazim and benomyl. This corresponds to the larger $\mathrm{EC}_{50}$ of $5.61 \mu \mathrm{M}$ thiabendazole on $F$. graminearum. We concluded that the higher the inhibition ratios of the three benzimidazole compounds on $F$. graminearum tubulin polymerization, the lower the $\mathrm{EC}_{50}(\mu \mathrm{M})$. Combined with the results of fluorescence quenching ratios of the three benzimidazole compounds (carbendazim, benomyl, and thiabendazole) to $\beta_{2}$-tubulin, the main mechanism of action (although they have others) of the three benzimidazole compounds consisted of benzimidazole compounds primarily binding to the $\beta$-tubulin, then disturbing the microtubule dynamic (Davidse 1986; Jamieson et al. 2011; Thelingwani et al. 2009).

Understanding the nature of the interaction between benzimidazole compounds and $F$. graminearum tubulin is fundamental for the development of tubulin-specific anti- $F$. graminearum compounds with selective activity, appropriate pharmacokinetic properties, and low human toxicity. Most notably, the results of this study provide better understanding of the mechanism of action of benzimidazole compounds.

\section{ACKNOWLEDGMENTS}

This work was supported by Special Fund for Agro-scientific Research in the Public Interest (number 201303023).

\section{LITERATURE CITED}

Ansari, K. F., and Lal, C. 2009. Synthesis, physicochemical properties and antimicrobial activity of some new benzimidazole derivatives. Eur. J. Med. Chem. 44:4028-4033.

Blackhall, W. J., Drogemuller, M., Schnieder, T., and von Samson-Himmelstjerna, G. 2006. Expression of recombinant $\beta$-tubulin alleles from Cylicocyclus nassatus (Cyathostominae). Parasitol. Res. 99:687-693.

Bradford, M. M. 1976. A rapid and sensitive method for the quantitation of microgram quantities of protein utilizing the principle of protein-dye binding. Anal. Biochem. 72:248-254.

Brown, H. D., Matzuk, A. R., Ilves, I. R., Peterson, L. H., Harris, S. A., Sarett, L. H., Egerton, J. R., Yakstis, J. J., Campbell, W. C., and Cuckler, A. C. 1961. Antiparasitic drugs. IV. 2-(4'-thiazolyl)-benzimidazole, a new anthelmintic. J. Am. Chem. Soc. 83:1764-1765.

Castoldi, M., and Popov, A. V. 2003. Purification of brain tubulin through two cycles of polymerization-depolymerization in a high-molarity buffer. Protein Expr. Purif. 32:83-88.

Chen, C., Bi, C., Yu, J., Wang, J., Li, H., Luo, Q., and Zhou, M. 2008. Carbendazim-resistance and its molecular mechanism in Gibberella zeae. J. Plant Pathol. 90:S2.17-77.

Chen, C.-J., Yu, J.-J., Bi, C.-W., Zhang, Y.-N., Xu, J.-Q., Wang, J.-X., and Zhou, M.-G. 2009. Mutations in a $\beta$-tubulin confer resistance of Gibberella zeae to benzimidazole fungicides. Phytopathology 99:1403-1411.

Chen, Y., Wang, J., Zhou, M., Chen, C., and Yuan, S. 2007. Vegetative compatibility of Fusarium graminearum isolates and genetic study on their carbendazim-resistance recombination in China. Phytopathology 97:1584-1589. 
Chiba, M., and Cherniak, E. A. 1978. Kinetic study of reversible conversion of methyl 1-(butylcarbamoyl)-2-benzimidazole carbamate (benomyl) to methyl 2-benzimidazole carbamate (MBC) and butyl isocyanate (BIC) in organic solvents. J. Agric. Food Chem. 26:573-576.

Clemons, G. P., and Sisler, H. D. 1971. Localization of the site of action of a fungitoxic benomyl derivative. Pestic. Biochem. Physiol. 1:32-43.

Davidse, L. C. 1973. Antimitotic activity of methyl benzimidazol-2-yl carbamate (MBC) in Aspergillus nidulans. Pestic. Biochem. Physiol. 3:317-325.

Davidse, L. C. 1975. Mode of action of methyl benzimidazol-2-yl-carbamate (MBC) and some biochemical aspects of acquired resistance against this fungicide in Aspergillus nidulans. In System Fungizide. Akademie Verlag, Berlin.

Davidse, L. C., and Flach, W. 1977. Differential binding of methyl benzimidazol2-yl carbamate to fungal tubulin as a mechanism of resistance to this antimitotic agent in mutant strains of Aspergillus nidulans. J. Cell Biol. 72:174-193.

Davidse, L. C. 1986. Benzimidazole fungicides: Mechanism of action and biological impact. Annu. Rev. Phytopathol. 24:43-65.

Davidse, L. C. 1987. Biochemical aspects of benzimidazole fungicides-action and resistance. Pages 245-257 in: Modern Selective Fungicides-Properties, Applications, Mechanisms of Action. H. Lyr, ed. Longman, London.

Delp, C., and Klopping, H. L. 1968. Performance attributes of a new fungicide and mite ovicide candidate. Plant Dis. Rep. 52:95-99.

Dong, H., Li, Y.-Z., and Hu, W. 2004. Analysis of purified tubulin in high concentration of glutamate for application in high throughput screening for microtubule-stabilizing agents. Assay Drug Dev. Technol. 2:621-628.

Eftink, M. R., and Ghiron, C. A. 1981. Fluorescence quenching studies with proteins. Anal. Biochem. 114:199-227.

Fourest-Lieuvin, A. 2006. Purification of tubulin from limited volumes of cultured cells. Protein Expr. Purif. 45:183-190.

Friedman, P. A., and Platzer, E. G. 1978. Interaction of anthelmintic benzimidazoles and benzimidazole derivatives with bovine brain tubulin. BBAGen. Subjects. 544:605-614.

Giles, N. L., Armson, A., and Reid, S. A. 2009. Characterization of trifluralin binding with recombinant tubulin from Trypanosoma brucei. Parasitol. Res. 104:893-903.

Goswami, R. S., and Kistler, H. C. 2004. Heading for disaster: Fusarium graminearum on cereal crops. Mol. Plant Pathol. 5:515-525.

Hammerschlag, R. S., and Sisler, H. D. 1973. Benomyl and methyl-2benzimidazole carbamate (MBC): Biochemical, cytological and chemical aspects of toxicity to Ustilago maydis and Saccharomyces cerevisiae. Pestic. Biochem. Physiol. 3:42-54.

Hoebeke, J., Van Nijen, G., and De Brabander, M. 1976. Interaction of oncodazole (R17934), a new anti-tumoral drug, with rat brain tubulin. Biochem. Biophys. Res. Commun. 69:319-324.

Hollomon, D. W., Butters, J. A., Barker, H., and Hall, L. 1998. Fungal betatubulin, expressed as a fusion protein, binds benzimidazole and phenylcarbamate fungicides. Antimicrob. Agents Ch. 42:2171-2173.

Hosamani, K. M., and Shingalapur, R. V. 2011. Synthesis of 2-mercaptobenzimidazole serivatives as potential anti-microbial and cytotoxic agents. Arch. Pharm. (WeinHeim) 344:311-319.

Howard, R. J., and Aist, J. R. 1977. Effects of MBC on hyphal tip organization, growth, and mitosis of Fusarium acuminatum, and their antagonism by $\mathrm{D}_{2} \mathrm{O}$. Protoplasma 92:195-210.

Howard, R. J., and Aist, J. R. 1980. Cytoplasmic microtubules and fungal morphogenesis: Ultrastructural effects of methyl benzimidazole-2-ylcarbamate determined by freeze-substitution of hyphal tip cells. J. Cell Biol. 87:55-64.

Jamieson, J. D., Smith, E. B., Dalvie, D. K., Stevens, G. J., and Yanochko, G. M. 2011. Myeloperoxidase-mediated bioactivation of 5-hydroxythiabendazole: A possible mechanism of thiabendazole toxicity. Toxicol. In Vitro 25: $1061-1066$

Jang, M.-H., Kim, J., Kalme, S., Han, J.-W., Yoo, H.-S., Kim, J., Koo, B.-S., Kim, S.-K., and Yoon, M.-Y. 2008. Cloning, purification, and polymerization of Capsicum annuum recombinant $\alpha$ and $\beta$ tubulin. Biosci. Biotechnol. Biochem. 72:1048-1055.

Koo, B.-S., Jang, M.-H., Park, H., Kalme, S., Park, H.-Y., Han, J.-W., Yeo, Y.-S., Yoon, S.-H., Kim, S.-J., Lee, C.-M., and Yoon, M.-Y. 2010. Characterization of Capsicum annuum recombinant $\alpha$-and $\beta$-tubulin. Appl. Biochem. Biotechnol. 160:122-128.
Koo, B., Kalme, S., Yeo, S., Lee, S., and Yoon, M. 2009a. Molecular cloning and biochemical characterization of $\alpha$-and $\beta$-tubulin from potato plants (Solanum tuberosum L.). Plant Physiol. Biochem. 47:761-768.

Koo, B.-S., Park, H., Kalme, S., Park, H.-Y., Han, J.-W., Yeo, Y.-S., Yoon, S.-H., Kim, S.-J., Lee, C.-M., and Yoon, M.-Y. 2009b. $\alpha$ - and $\beta$-tubulin from Phytophthora capsici KACC 40483: Molecular cloning, biochemical characterization, and antimicrotubule screening. Appl. Microbiol. Biotechnol. 82:513-524.

Lubega, G. W., Geary, T. G., Klein, R. D., and Prichard, R. K. 1993. Expression of cloned $\beta$-tubulin genes of Haemonchus contortus in Escherichia coli: Interaction of recombinant $\beta$-tubulin with native tubulin and mebendazole. Mol. Biochem. Parasitol. 62:281-292.

MacDonald, L. M., Armson, A., Thompson, R. A., and Reynoldson, J. A. 2004. Characterisation of benzimidazole binding with recombinant tubulin from Giardia duodenalis, Encephalitozoon intestinalis, and Cryptosporidium parvum. Mol. Biochem. Parasitol. 138:89-96.

Mertens, M. L., and Kägi, J. H. 1979. A graphical correction procedure for inner filter effect in fluorescence quenching titrations. Anal. Biochem. 96: 448-455.

Minoura, I., Hachikubo, Y., Yamakita, Y., Takazaki, H., Ayukawa, R., Uchimura, S., and Muto, E. 2013. Overexpression, purification, and functional analysis of recombinant human tubulin dimer. FEBS Lett. 587: 3450-3455.

Navarrete-Vázquez, G., Rojano-Vilchis, M. de M., Yépez-Mulia, L., Meléndez, V., Gerena, L., Hernández-Campos, A., Castillo, R., and Hernández-Luis, F. 2006. Synthesis and antiprotozoal activity of some 2(trifluoromethyl)-1H-benzimidazole bioisosteres. Eur. J. Med. Chem. 41: 135-141.

Oxberry, M. E., Geary, T. G., Winterrowd, C. A., and Prichard, R. K. 2001. Individual expression of recombinant $\alpha$-and $\beta$-tubulin from Haemonchus contortus: Polymerization and drug effects. Protein Expr. Purif. 21:30-39.

Qiu, J., Xu, J., Yu, J., Bi, C., Chen, C., and Zhou, M. 2011. Localisation of the benzimidazole fungicide binding site of Gibberella zeae $\beta 2$-tubulin studied by site-directed mutagenesis. Pest Manage. Sci. 67:191-198.

Roy, A., Kucukural, A. and Zhang, Y. 2010. I-TASSER: A unified platform for automated protein structure and function prediction. Nat. Protoc. 5:725-738.

Sackett, D. L., and Varma, J. K. 1993. Molecular mechanism of colchicine action: Induced local unfolding of .beta.-tubulin. Biochemistry 32: 13560-13565

Staron, T., Allard, X., and Gug, M. 1964. Propriétés antifongiques des 2-(4'thiazolyle) benzimidazole ou thiabendazoles. Phytiatrie Phytopharm. 13: 163-168.

Tang, C., Yanagihara, K., and Zhang, Y. 1992. 1-butyl isocyanate from aqueous Benlate ${ }^{\circledR}$ formulations. Arch. Environ. Contam. Toxicol. 23: $270-272$

Thelingwani, R. S., Zvada, S. P., Dolgos, H., Ungell, A. B., and Masimirembwa, C. M. 2009. In vitro and in silico identification and characterization of thiabendazole as a mechanism-based inhibitor of CYP1A2 and simulation of possible pharmacokinetic drug-drug interactions. Drug Metab. Dispos. 37:1286-1294.

Tunçbilek, M., Kiper, T., and Altanlar, N. 2009. Synthesis and in vitro antimicrobial activity of some novel substituted benzimidazole derivatives having potent activity against MRSA. Eur. J. Med. Chem. 44:1024-1033.

Twu, J. S., and Wold, F. 1973. Butyl isocyanate, an active-site-specific reagent for yeast alcohol dehydrogenase. Biochemistry 12:381-386.

Uppuluri, S., Knipling, L., Sackett, D. L., and Wolff, J. 1993. Localization of the colchicine-binding site of tubulin. Proc. Nat. Acad. Sci. USA 90: 11598-11602.

Widlund, P. O., Podolski, M., Reber, S., Alper, J., Storch, M., Hyman, A. A., and Drechsel, D. N. 2012. One-step purification of assemblycompetent tubulin from diverse eukaryotic sources. Mol. Biol. Cell 23: 4393-4401.

Yoon, Y., and Oakley, B. R. 1995. Purification and characterization of assembly-competent tubulin from Aspergillus nidulans. Biochemistry 34: 6373-6381.

Zhang, Y. 2008. I-TASSER server for protein 3D structure prediction. BMC Bioinf. 9:40 